\title{
History of the HIV Epidemic in China
}

\section{Zunyou Wu ${ }^{1,2} \cdot$ Junfang $C^{2}{ }^{3} \cdot$ Sarah Robbins Scott ${ }^{1}$ - Jennifer M. McGoogan ${ }^{1}$}

Published online: 26 November 2019

(C) Springer Science+Business Media, LLC, part of Springer Nature 2019

\begin{abstract}
Purpose of Review This study aims to review the history of the human immunodeficiency virus (HIV) infection epidemic in China.

Recent Findings The HIV infection epidemic in China has evolved significantly over the past 35 years, from initially exclusively within people who inject drugs (PWID), to outbreaks due to plasma collection contamination in the mid-1990s, to now almost exclusive transmission via sexual contact. The number of newly-diagnosed cases and the number HIV-related deaths have increased each year since 2004, coinciding with a massive scale-up of both HIV testing and antiretroviral therapy initiation. The proportion of cases diagnosed later in their disease progression has remained constant.

Summary The initial outbreaks of HIV across China were identified quickly and the overall trends have been monitored. While the HIV epidemic among PWID has been well managed, the growing HIV epidemic via sexual contact has grown more complex and even more difficult to control.
\end{abstract}

Keywords Human immunodeficiency virus infection $\cdot$ Case reporting $\cdot$ China $\cdot$ Control $\cdot$ Diagnosis $\cdot$ Epidemic $\cdot$ Prevalence Surveillance $\cdot$ Transmission

\section{Introduction}

The human immunodeficiency virus (HIV) epidemic in China began later and developed slower compared to other countries across the globe, including those in Asia. Since the first reported cases in China, efforts have been made to reduce the transmission rate and disease burden of HIV as quickly as possible and to monitor ongoing epidemic trends across the country (1). China has made great progress in its HIV response over the past two decades, adopting key legislation and policies aimed at improving the health and well-being of people living with HIV (PLWH) and providing prevention services for individuals and communities $(2,3 \bullet \bullet)$.

This article is part of the Topical Collection on The Global Epidemic

Zunyou Wu

wuzy@263.net

1 National Center for AIDS/STD Control and Prevention, Chinese Center for Disease Control and Prevention, 155 Changbai Road, Changping District, Beijing 102206, China

2 University of California, California, Los Angeles, USA

3 Hangzhou Center for Disease Control and Prevention, Hangzhou, China
In 2003, the Chinese government announced its "Four Frees and One Care" policy, which provided free antiretroviral therapy (ART) to all PLWH, free voluntary counseling and testing, free prevention of mother-to-child transmission (PMTCT) services, free schooling for children orphaned or otherwise affected by HIV or acquired immune deficiency syndrome (AIDS), and economic assistance to households of PLWH (4). This policy has significantly eased the health and economic burden associated with HIV infection, increased HIV screening and linkage to care, and improved overall health of PLWH. More so, this foundational policy focused China's leadership on an HIV response strategy characterized by critical evaluation of current evidence, pragmatic development of tailored interventions, and iterative assessment and improvement of HIV-related services. For example, the World Health Organization (WHO) has repeatedly revised treatment guidelines for resource-limited settings, and each time, leaders of China's National Free ART Program have reviewed the evidence and expanded ART coverage to meet WHO-recommendations. Most recently, in 2016, CD4-based ART eligibility requirements were eliminated in China (5), and in the following year, almost 600,000 PLWH in China were accessing ART (6).

Epidemiological studies and surveillance programs have been crucial to the development and scale-up of HIV response 
policies, documenting the evolution of HIV in China including outbreaks among key populations such as people who inject drugs (PWID), former plasma donors (FPD), and men who have sex with men (MSM), and evaluating response effectiveness (1). Effective data collection, management, and analysis are necessary for the development of comprehensive response strategies and are essential to timely identification of outbreaks. In 2006, the National Center for AIDS/STD Control and Prevention (NCAIDS) at the Chinese Center for Disease Control and Prevention (China CDC) launched its national, web-based HIV/AIDS information system, the Comprehensive Response Information Management System (CRIMS) (7). This system has improved the efficiency of data collection and analysis and is a key tool for the implementation of national HIV/AIDS programs in China and is an important resource for epidemiological study since today it tracks the largest cohort of PLWH in the world.

However, regardless of these efforts, China is still home to one of the largest HIV epidemics in the world, with a continually growing number of new cases identified each year. This paper provides a brief history of the HIV epidemic and highlights a few aspects of China's HIV/AIDS response.

\section{Detection of HIV Outbreaks}

\section{Outbreak among People Living with Hemophilia}

After AIDS was first reported in the United States in the early 1980s, Chinese scientists began to search for possible cases in China. In 1984, Zeng and colleagues collected 380 blood specimens from both healthy people and patients seeking care at various health facilities in eight provinces throughout China, but no HIV cases were found. In 1985, Zeng and colleagues collected blood specimens from 28 hemophilia patents and discovered that four were HIV-reactive, and that they had received contaminated factor VIII imported from United States (8). These four patients became the first reported cases of HIV infection in China. All other cases identified in China up to 1989 were among foreign visitors or Chinese nationals returning from overseas $(9,10)$.

\section{Outbreak among People Who Inject Drugs}

In October 1989 in detoxification centers in Ruili County, Yunnan Province, a rural area in southwestern China bordering Myanmar, Ma and colleagues collected blood specimens from 175 drug users in order to screen for viral hepatitis infection, which was known to be spreading through sharing of heroin injecting equipment at that time. They also tested the residual specimens for HIV infection and identified 79 cases, for an HIV prevalence of $45 \%$ (11). This study provided the first evidence of the first major HIV outbreak among PWID in China.

Between April 1989 and April 1990, Ma and others expanded their initial survey to other populations including healthcare workers, patients seeking care at health facilities, and blood donors. A total 2530 blood specimens were collected during this time, 309 of which were found to be HIV-reactive, for a prevalence of $23 \%$ among PWID, $3 \%$ among sexual partners of PWID, and $13 \%$ among immigrants from Myanmar. These results suggested that new HIV cases were still limited to PWID and their partners and had not yet expanded to other high-risk groups or to the general population (12).

In order to assess the timing and the geography of the outbreak, Chinese researchers used historical specimens collected from three different time periods (1986-1988, 1989, and 1990), different geographic areas, and different populations. A total 17,021 specimens were tested, and $429 \mathrm{HIV}$ cases were found. Importantly, the results of this study showed that the newly diagnosed HIV infections were limited to specimens collected in and after 1989, in Dehong Prefecture, among PWID (13). These findings supported the conclusion that the HIV outbreak among PWID was identified in a timely fashion.

\section{Outbreak among Former Plasma Donors}

Between late 1994 and early 1996, HIV outbreaks were reported among former plasma donors (FPD) in poor rural communities across several provinces in central and eastern China $(4,14)$. In response to the small outbreak among people living with hemophilia who had received contaminated factor VIII from the United States, the Chinese government banned the importation of blood products. This abrupt decline in imported blood supply suddenly created high demand for domestic blood products. However, due to traditional Chinese cultural beliefs, voluntary blood donation was rare (14). As a result, many small, commercial and government-managed plasma collection stations, which paid people to donate blood, were set up primarily in rural areas in China throughout the early 1990s $(14,15)$. Unfortunately, the leadership of many of these stations placed the pursuit of profits ahead of the protection of patients and unsafe blood product collection practices caused an outbreak of HIV infection among FPD (15).

In 1994, according to a Shanghai pharmaceutical company's reports, the plasma donated by a woman in Anhui province, eastern China, repeatedly tested HIV positive. Upon further investigation, it was discovered that her two daughters also had HIV, but that neither her son nor her husband did. When it was determined that all three women had engaged in plasma donation, the contamination created by dangerous blood collection and processing practices was discovered (16). Further testing of previously collected plasma in 
storage showed an unbelievably high proportion of donors had HIV infection $(17,18)$.

Around the same time, in the northern city of Tianjin, a blood donor from Hebei province also tested positive for HIV at the Tianjin Blood Bank. Surveys of commercial blood donors in the province found an HIV prevalence of 4\% (17). However, testing of residual blood specimens collected during a hepatitis study conducted in 1994 found no HIV, suggesting that the introduction of HIV into the blood collection system began in late 1994.

\section{Outbreak among Men Who Have Sex with Men}

Unlike in many other countries where the HIV epidemic started among MSM, very few HIV cases had been reported in MSM even up until 2005. However, the rate of HIV has been increasingly drastically ever since. While the prevalence was reported to be $1.4 \%$ in 2005 (19), a national survey carried out in 61 cities throughout China from 2008 to 2009 established a national-level HIV prevalence of 5\% among MSM in China (20). This has continued to climb up to $8 \%$ in 2015 (19). However, single-city prevalence results in many cases were much higher.

More recently, the estimated national HIV prevalence among MSM has reached 6.9\% (21). The proportion of newly diagnosed MSM cases increased from $0.3 \%$ in 2005 to $12 \%$ in 2010 , peaking at $28.2 \%$ in 2015 , then slightly declining to $23.3 \%$ in 2018 (21). Since 2010, the prevalence in MSM is the highest infection rate among all key populations in China (22•).

\section{Nosocomial Outbreak among Women in Treatment for Recurrent Miscarriage}

Though the previous sections have discussed the historical identification of HIV cases and outbreaks of the disease among different populations, a recent isolated event occurred in Hangzhou, China linked to nosocomial transmission of the disease. In January 2017, an HIV outbreak was reported at a hospital in the southern Chinese city, due to lymphocyte immunotherapy (LIT) performed at the hospital in the prior year (23). The index patient's exposure was traced to her husband, who reportedly had condomless, receptive anal sex with a man prior to LIT donation (23). Additionally, a failure to follow the official laboratory protocol by the attending technician led to unsafe exposure to HIV. It was identified that, due to a lack of disposable sterile equipment at the hospital, the attending healthcare worker reused LIT tubes, likely leading to a number of LIT patients receiving the contaminated lymphocytes from the infected, index case-patient (23). All LIT services were immediately suspended until new guidelines were issued in late December 2017, restricting the further spread of the outbreak.
HIV Epidemics by Mode of Transmission

\section{Transmission Via Blood Product Contamination}

The outbreak of HIV infection among FPD around the mid1990 s took the nation by complete surprise. Limited epidemiological investigations suggested that the outbreak actually took place from late 1994 to early 1996. It was believed that thousands and thousands of plasma donors and blood recipients had been infected. One survey conducted in a rural area in 1996 indicated that the HIV prevalence among former commercial plasma donors was $12.5 \%$ (24). Unfortunately, due to the sensitive nature of the topic, no further national surveys were conducted at that time to understand the magnitude of the HIV epidemic among commercial blood plasma donors in China.

Sporadic epidemiological surveys with small sample size revealed high rates of HIV infection among former plasma donors. For example, among 96 donors who engaged in underground plasma donation in 1998-1999, 74\% tested positive for HIV infection (25).

In response, China enacted its first blood safety law in 1998 (4). In this law, blood donation was permitted only if voluntary and repeated commercial donation was prohibited (4). Additionally, government officials realized that blood donors whom were most likely infected might be reluctant to get tested for HIV, particularly as there was no treatment available in China at that time. Because of severe stigma and low awareness of HIV/AIDS in Chinese society at this time, the Chinese government announced the "Four Frees and One Care" AIDS policies (4).

Even though there were supportive policies, no former plasma or blood donors came for HIV screening, therefore, no HIV infection via plasma donation or blood transfusion was reported. It was evident that if no one was tested for HIV, then there would be no HIV infection diagnosed or reported. Thus, the Ministry of Health launched a national HIV testing campaign in 2004-2005, specifically targeting former commercial plasma donors who had donated blood or plasma in the mid-1990s. During this campaign, each province searched for FPD and encouraged them to participate in HIV screening. Each province was also provided an option whether or not to include other key populations such as PWID, MSM, sex workers, and patients attending sexually transmitted diseases (STD) clinics. The HIV testing campaign was held from July 2004 to June 2005 across China. During this 12-month HIV period, close to one million former plasma donors were tested for HIV and about 23,000 were diagnosed. The prevalence of HIV infection among former commercial plasma donors was $2.3 \%$.

The prevalence of HIV infection among former plasma donors from the national HIV testing campaign was much lower than expected. Just before the testing campaign in 
2003, one scientific epidemiological study among former plasma donors conducted in Shanxi found a similar result. The sample was big enough to have provided a valid estimation of HIV prevalence. Among 3062 participating villagers, $29.5 \%$ reported a history of selling whole blood or plasma, and HIV prevalence was estimated to be $1.3 \%$ overall and $4.1 \%$ among plasma donors (26). Again, it was much lower than the previously thought.

It was not until the national HIV testing campaign among former commercial blood plasma donors in 2004 and 2005 that the true magnitude of the HIV outbreak among FPD became known - an estimated 69,000 HIV infections were caused by contamination during blood plasma collection or transfusion of contaminated blood products in China during the mid-1990s (27).

After this HIV testing campaign, many believed that most of those individuals infected via blood or plasma contamination had been screened and diagnosed and the residual number of donors infected would be limited. However, the number of newly diagnosed HIV positive individuals reporting their route of HIV transmission via blood or plasma contamination remained unacceptably high in the subsequent years, for example, over 7000 newly diagnosed individuals in 2006, 2007, and 2008. Since this time however, the number of newly diagnosed HIV infections due to blood or plasma contamination has fallen almost to zero. By 2015, almost 20 years after the initial HIV outbreak, no cases due to blood or plasma contamination have been reported.

Of note however is the "window period" of infection, or the 2 to 3 weeks' time between the onset of HIV infection and the appearance of detectable antibodies. During this time, blood product recipients may be infected via contaminated blood transfusion. In order to reduce this period of infectiousness and capture the true number of positive cases, since 2015, the Chinese government has provided ribonucleic acid (RNA) screening for all blood banks. Due to the introduction of these new testing measures, the "window period" has been shortened to 7 to 11 days, thus reducing the potential risk of HIV infection.

\section{Transmission Via Injection Drug Use}

Since the initial cases of HIV were identified among PWID in Ruili, Yunnan, in 1989, the majority of HIV infections in the early phase of the epidemic were found to be among drug users in this region only. Yunnan in Southern China, located along a drug trafficking route known as the "Golden Triangle," is a major entry point of opiates and other drugs from the China/Myanmar border (28). About a third of drugs produced in this area are reportedly trafficked through China (29). Subsequently, the increased availability of drugs in this region led to drastic increases in drug abuse. Thus, the introduction of drug use in this region created an opportunity for
HIV infections to flourish, due to unsafe sexual behaviors and sharing of injection equipment $(28,29)$. During this time, the prevalence of HIV infection among PWID was found to be as high as $80 \%$ or more in some areas of Yunnan Province (30).

In 1995, new cases of HIV were reported among PWID in Sichuan and Xinjiang Provinces. From there, the HIV epidemic among PWID spread rapidly, particularly along the drug trafficking routes along the China/Myanmar border, and then to the rest of China. By 2002, all provinces in China reported HIV infection among PWID. It took less than 15 years for the virus to spread from the initial infections in Yunnan to across all provinces in the country; from rural areas to urban areas; and from border communities to central parts of the country (28).

The HIV prevalence and number of newly diagnosed HIV cases in PWID peaked from 2003 to 2005. Since that time, HIV in PWID has generally declined across all provinces. The national prevalence of HIV infection among drug users declined from $7.5 \%$ in 2005 to $2 \%$ in 2018 (21). The number of newly diagnosed HIV cases among drug users declined from almost 20,000 cases per year in 2005 to less than 4000 per year in 2018 (21).

This steady decline of HIV infections among PWID is likely due to nationwide scale-up of harm reduction programs, including methadone maintenance treatment (MMT) and needle and syringe exchange programs (NSP) (31). MMT for PWID was first introduced in early 2004 in Sichuan, Zhejiang, Guangxi, Yunnang, and Guizhou provinces and expanded to more than 30 MMT clinics across 11 provinces by 2005 (29). The results of this pilot program were then used to develop national policies on NSP and MMT (32), where now, more than 760 MMT clinics serving some 150,000 clients and 700 NSP sites serving 40,000 PWID annually exist (21). The use of MMT and NSP programs have become a cornerstone of China's HIV response, drastically reducing the epidemic among drug users in the country. Studies have reported that needle-sharing behaviors among PWID have lessened since the introduction of these programs (33). Ensuring PWID are retained in such programs will be a continued challenge for the control of the epidemic.

\section{Transmission Via Male-Male Sexual Contact}

The sexual route of HIV transmission now exceeds that of injection drug use in China, with $95 \%$ of cases reportedly due to sexual transmission (34). This is particularly reflected in the MSM population, where the male-male sexual contact route of HIV transmission is on the rise. HIV incidence and prevalence is higher than any other high-risk population in China, and continues to increase, particularly in larger, urban areas $(22 \bullet, 35)$. Though male-male sex has been legal in China since 1997, sociocultural factors mean this group often faces stigma and discrimination, causing them to hide their sexual 
behaviors from partners and medical workers (36). The importance of family in Chinese culture also places substantial pressure on MSM to conceal their MSM identity (37). Subsequently, HIV prevention and testing rates in MSM have reportedly remained low, most likely due to fears of disclosing personal information or being discriminated against (37). Additionally, recreational drug and alcohol use in MSM in China has also been associated with unsafe sexual behaviors, particularly unprotected anal and receptive sex and multiple sexual partners (38).

In response, the government has taken strides in order to prevent ongoing HIV transmission among MSM, developing national guidelines for the control of HIV in this high-risk group (32). Additionally, China is providing innovative strategies to HIV testing for MSM in cooperation with community based organizations (CBOs), including assessing the feasibility and acceptability of HIV self-testing, including testing kits in vending machines on college campuses, and using social networking applications to provide HIV counseling and testing (38). Specifically, in combination with public health agencies and CBOs, innovative efforts to improve care through the use of the Internet and Wechat have been adopted (38). Additional scale up of condom promotion activities and outreach services using new and innovative techniques such as social networking applications are a few of the key strategies being implemented to reduce HIV among $\operatorname{MSM}(38,39)$. Despite these efforts however, the prevalence continues to rise. New interventions, such as the rollout of pre-exposure prophylaxis (PrEP) in this group are currently be considered.

\section{Transmission Via Heterosexual Contact}

The number of newly diagnosed HIV infections due to heterosexual transmission surpassed the number of cases due to injection drug use for first time in 2007, 19 years after the first outbreak of HIV among PWID in 1989 (40•). Since then, the proportion of HIV cases due to heterosexual contact has continually increased, from $50 \%$ in 2010 , to over $60 \%$ in 2011 , and more than $70 \%$ in 2018 (21). Similarly, the number of new infections in HIV-serodiscordant couples has also increased (41). One study of sero-discordant couples in Yunnan reported that the risk of HIV was reduced by one third in uninfected partners of PLWH receiving ART compared to those not receiving ART (41). After implementation of treatment as prevention among sero-discordant couples, the HIV incidence via marital sexual contact has significantly declined, from $2.6 \%$ in 2011 to $1.1 \%$ in 2018 (21).

The prevalence of HIV infection among a few key populations who are believed to be at high risk of acquiring HIV infection through heterosexual contact has remained consistently low. For example, the HIV prevalence in female sex workers has remained under $0.5 \%$ since $1995(21,42)$, while the prevalence of HIV among male STD clinic attendees has also remained low $(21,42)$.

\section{Transmission among Transgender Persons}

Limited information on HIV infection in transgender groups in China is available. A recent study found that HIV infection among transgender women reached $12.4 \%$ in Shanghai, but $3.4 \%$ in Tianjin (43). An additional study found rates of HIV testing among transgender individuals to be substantially lower than rates reported in other groups, most likely due to stigma and a lack of community support (44). Among transgender women in China, it was found that those who had casual partners were more likely to engage in high-risk behaviors because of a lack of HIV disclosure and limited knowledge regarding prevention tactics (45). Further research is needed which aims to identify the key drivers of HIV among this at-risk group and to understand the unique barriers to care transgender populations face throughout China.

\section{Mother-to-Child Transmission (MTCT)}

HIV prevalence among pregnant women in China who accessed antenatal care (ANC) is quite low, at below $0.1 \%$ (46). Controlling the HIV epidemic in pregnant women and reducimg mother-to-child transmission greatly depends on the coverage of prevention of mother-to-child transmission (PMTCT) programs. HIV testing coverage in pregnant women attending ANC and in HIV-exposed infants at 18 months has significantly increased, from $62.4 \%$ and $22.1 \%$ in 2003 to $90.3 \%$ and $82.8 \%$ in 2011, respectively (46).

National sentinel surveillance data suggested that the prevalence of HIV infection among pregnant women was 19.8/ 100,000 in 2011 and decreased slightly, to $9.1 / 100,000$ in 2016. A recent study reported that among 15 million pregnant women screened for HIV infection in China, an overall prevalence of 34.0 per 100,000 was found (47). The prevalence among pregnant women was found to be higher for those living in Southern China compared to those residing in Northern China, with the highest prevalence in Western China, at 93.5 cases per 100,000 persons. The lowest prevalence among pregnant women was in Eastern Beijing, which reported 8.6 cases per 100,000 persons (47). This means that the HIV prevalence in Western China was 11 times higher than in Eastern China. Among 31 provinces, six had a prevalence of more than 50.0 cases per 100,000 persons, including Yunnan, Xinjiang, Sichuan, Guangxi, Guizhou and Chongqing. The number of pregnant women in these 6 provinces accounted for $21 \%$ of all pregnant women in the country, but the number of HIV cases accounted for $76 \%$ of all HIV cases diagnosed among pregnant women in mainland China. Further analysis showed that 30 cities reported an HIV 
prevalence among pregnant women of more than 100.0 cases per 100,000 persons, 28 of which were located in the Southwest of China (47).

In 2005, China started its first PMTCT program in eight cities and in 2015, launched its Integrated Prevention of Mother-toChild Transmission of HIV, Syphilis, and Hepatitis B program (iPMTCT Program) nationwide (48). As a result, access to care and treatment for HIV positive mothers and their children has significantly improved. ART prophylaxis uptake among HIV infected pregnant women and their infants has increased from $35.2 \%$ and $26.9 \%$ in 2003 to $86.2 \%$ and $90.3 \%$ in 2011, respectively (46). The HIV vertical transmission rate has substantially decreased, from $31.8 \%$ prior to the initiation of PMTCT programs, to 2.3\% in 2011 (46). Scale-up of PMTCT programs are a key priority for the current management of HIV among pregnant women and their children.

\section{Geographic Distribution of HIV Cases}

Though overall HIV infection is now driven by sexual contact, particularly heterosexual contact, the distribution of HIV cases varies significantly across China. The initial phase of the HIV epidemic was primarily concentrated in rural areas in Southwestern and Western China as seen in Fig. 1a, then spreading into cities and urban areas across all provinces $(9$, 39). According to sentinel surveillance data, in 1998, more than 1000 cases were identified in Yunnan and Xinjiang Province, while the remaining provinces reported far fewer cases. As previously discussed, these new infections did not remain contained in just southwestern China. On the contrary, due to the increase in drug use and unsafe sexual behaviors, the epidemic began to spread and by the next decade, was present in all provinces throughout the country. The largest number of PLWH continued to be located in Southern and
Southwestern China, though a growing number of cases were identified in Central China, as seen in Fig. 1b. Yunnan was still home to the largest epidemic at the time, with more 100,000 PLWH, followed by Xinjiang, Sichuan, Guangxi, Guangdong, and Henan, which reported more than 50,000 cases in 2008. The eastern coast of China also began to experience a growing number of new cases, with many provinces in the region reporting more than 10,000 cases (Fig. 1b). This trend has continued to today, where in 2018, Southwestern China still reports the largest number of HIV cases. Yunnan and Sichuan had more than 100,000 PLWH in 2018, followed by Guanxi, Guangdong, and Henan with more than 50,000 cases in the same year (Fig. 1c). The epidemic has continued to grow in Eastern and Northern China, while regions such as Inner Mongolia, Qinhai, Hainan, and Tibet report the fewest number of PLWH, with less than 5000 in 2018.

These epidemic patterns can be divided into two broad categories. The HIV epidemic has transitioned from injection drug using (as seen in the initial epidemic in South and Southwestern China) into heterosexual transmission. From 1989 to 1995 , majority of HIV infections were due to injecting drug use (49). Now, however, over $70 \%$ of newly diagnosed HIV cases are due to heterosexual contact (21). Currently, male-male sexual contact is the major mode of HIV transmission in China, particularly in larger, urban cities, such as in Beijing, Shanghai, Tianjin, as well as other parts of Northern China, as seen in Fig. 1c.

\section{HIV/AIDS Compared to Other Infectious Diseases}

After the Severe Acute Respiratory Syndrome (SARS) outbreak in 2003, the mortality caused by all infectious diseases in China increased steadily, though most were attributed to HIV/AIDS
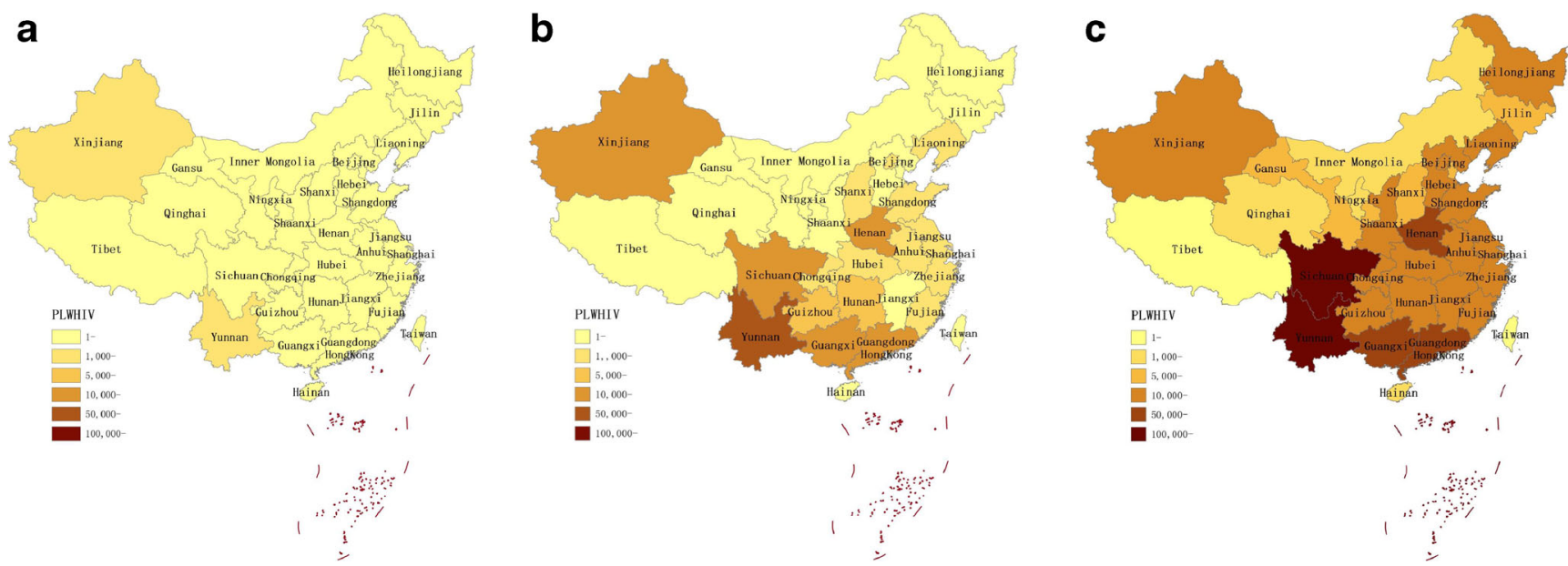

Fig. 1 a Geographic distribution of PLWH in China in 1998, b Geographic distribution of PLWH in China in 2008, (c) Geographic distribution of PLWH in China in 2018 
(50). Even with the free provision of ART to all infected patients in China, the number of deaths reported among PLWH and the proportion of overall mortality due to HIV/AIDS has steadily increased. In 2003, the total number of deaths caused by all infectious diseases combined was 6474 , while 379 of which were due to AIDS, being the fifth leading cause of death among all notifiable diseases that year (51). In 2008, the number of AIDSrelated deaths increased to 5389 and became the leading cause of death among all infectious diseases for the first time (52). In 2018, the number of AIDS-related deaths increased to 18,780 which was 4.3 times all other infectious disease related deaths combined (53). Previous research in China has shown multiple factors contributing to this rise in HIV/AIDS related mortality. In particular, late diagnosis, education level, socioeconomic status, and older age were also associated with AIDS-related mortality (54). Thus, earlier diagnosis and immediate ART initiation are strategies that could be strengthened to reduce this burden of HIV-related deaths.

\section{Conclusions}

Over the past 35 years, the HIV epidemic in China has evolved remarkably. China has implemented countless laws, policies, and guidelines to support the HIV/AIDS response, committing both political and economic will to the fight against HIV/AIDS. Based on the available epidemiological data, the HIV/AIDS response has been drastically scaled up across the country, increasing access to services and ultimately reducing the prevalence of the disease. What initially began as an epidemic among injection drug users has now shifted to heterosexual transmission in the general population. After the implementation of harm reduction strategies in 2004, the HIV epidemic in PWID has steadily declined, while HIV transmission via sexual contact continues to increase steadily and slowly, now accounting for $95 \%$ of newly reported cases. Though the prevalence of HIV infection has remained low in female sex workers and male STD clinic attendees, the number of HIV cases caused by heterosexual transmission continues to increase. This, coupled with the rising number of deaths, remains challenges for controlling the HIV epidemic in China. Improving access to HIV testing and linking patients to care prior to their progression to AIDS should be a key focus of future strategies and evidence-based policies.

Authors Contributions ZW wrote the initial draft of the manuscript. ZW, JC, SRS, and JMM edited and revised the manuscript. All authors approved the final version for submission.

Funding Information This work was funded by China's National Health Commission (grant number 2018ZX10721102). The study sponsor had no role in the writing of the report and in the decision to submit the paper for publication. ZW had final responsibility for the decision to submit for publication.

\section{Compliance with Ethical Standards}

Competing Interests The authors declare no competing interests.

Human and Animal Rights and Informed Consent This article does not contain any studies with human or animal subjects performed by any of the authors.

Disclaimer The views and opinions expressed here belong to the authors alone, and do not necessarily reflect the official views or endorsement of their affiliated institutions.

\section{References}

Papers of particular interest, published recently, have been highlighted as:

- Of importance

•. Of major importance

1 Sun X, Wang N, Li D, Zheng X, Qu S, Wang L, et al. The development of HIV/AIDS surveillance in China. AIDS. 2007;21(Suppl 8):S33-S8.

$2 \mathrm{Wu}$ Z. Achievement of HIV/AIDS program in the past 30 years and challenges in China. Chinese Journal of Epidemiology. 2015;36(12):1329-31.

3•• Pisani E, Wu Z. HIV in China: 30 years in numbers. In: Wu Z, editor. HIV/AIDS in China- beyond the numbers (English). Singapore: People's Medical Publishing House, Springer; 2017. p. 119-37. This chapter provides detailed desription, in quantitative terms, of the achivements China has made in controlling its HIV/AIDS epidemic over the past three decades.

4. Sun X, Lu F, Wu Z, Poundstone K, Zeng G, Xu P, et al. Evolution of information-driven HIV/AIDS policies in China. International journal of epidemiology. 2010;39 Suppl 2:ii4-13.

5. Yang X, Li X, Qiao S, Zhang Q, Shen X, Zhou Y. Immunological and virologic outcomes of people living with HIV in Guanxi, China: 2012-2017. PLoS One. 2019;14(3):e0213205.

6. Zhang F, Ma Y. Progress and challenges in China's free ART programme. Lancet HIV 2019;6(1):PE8-E9.

7. Mao Y, Wu Z, Poundstone K, Wang C, Qin Q, Ma Y, et al. Development of a unified web-based national HIV/AIDS information system in China. International journal of epidemiology. 2010;39 Suppl 2:ii79-89.

8. Zeng Y, Fan J, Zhang Q, Wang P, Tang D, Zong S, et al. Detection of antibody to LAV/HTLV-III in sera from hemophiliacs in China. AIDS Research. 1986;2(Supplement 1):S147-9.

9. Jia Z, Wang L, Chen RY, Li D, Wang L, Qin Q, et al. Tracking the evolution of HIV/AIDS in China from 1989-2009 to inform future prevention and control efforts. PLoS One. 2011;6(10):e25671.

10. Zhang KL. Epidemiology of HIV in China- intravenous drug users, sex workers, and large mobile populations are high risk groups. BMJ. 2002;324(7341):803-4.

11. Ma Y, Li Z, Zhang K, Yang W, Ren X, Yang Y, et al. HIV infections were first time found among people who inject drugs in China. Chinese Journal of Epidemiology. 1990;11:184-5.

12. Zhang J, Cheng H, Zhao S, Yang W, Ma Y, LI R, et al. An epidemiological study on HIV infection in Ruili County. Yunnan Province Chinese Journal of Epidemiology. 1991;12(1):9-11.

13. Zhao S, Cheng H, Zhang J, Li Z, Ma Y, Kou J, et al. AIDS surveillance in Yunnan Province of China (1986-1990). Chinese Journal of Epidemiology. 1991;12(2):72-4. 
14. He N, Detels R. The HIV epidemic in China: history, response, and challenge. Cell Res. 2005;15(11-12):825-32.

15. Ji G, Detels R, Wu Z, Yin Y. Correlates of HIV infection among former blood/plasma donors in rural China. AIDS. 2006;20(4): 585-91.

16. Wu Z, Liu Z, Detels R. HIV-1 infection in commercial plasma donors in China. Lancet. 1995;346(8966):61-2.

17. Wu Z, Dong N, Guo W. Discovery and control of the HIV/AIDS epidemic among plasma donors in China. In: Li L, Zhan S, editors. Epidemiological research cases in China (English). Beijing: People's Medical Publishing House; 2008. p. 153-64.

18. Chaddah A, Wu Z. Selling blood spreads HIV. In: Wu Z, editor. HIV/AIDS in China- beyond the numbers (English). Singapore: People's Medical Publishing House, Springer; 2017. p. 9-22.

19. Ye M, Giri M. Prevalence and correlates of HIV infection among men who have sex with men: a multi-provincial cross-sectional study in the southwest of China. HIV AIDS (Auckl). 2018;10: $167-75$.

20. Wu Z, Xu J, Liu E, Mao Y, Xiao Y, Sun X, et al. HIV and syphilis prevalence among men who have sex with men: a cross-sectional survey of 61 cities in China. Clin Infect Dis. 2013;57(2):298-309.

21. National Center for AIDS/STD Control and Prevention, China CDC Annual Report of China National HIV/STD/HCV Comprehensive Prevention and Treatment Programs in 2018. Beijing: National Center for AIDS/STD control and prevention, China CDC; 2019.

22. Cui Y, Guo W, Li D, Wang L, Shi CX, Brookmeyer R, et al. Estimating HIV incidence among key affected populations in China from serial cross-sectional surveys in 2010-2014. J Int AIDS Soc. 2016;19(1):20609. This paper describes HIV prevalence among key poplations and gives an estimation of HIV incidence among commercial sex workers, people who inject drugs and men who have sex with men.

23. Pan X, Jiang J, Ma Q, Zhang J, Yang J, Chen W, et al. Outbreak of HIV infection linked to nosocomial transmission, China, 20162017. Emerg Infect Dis. 2018;24(12):2141-9.

24. Wu Z, Rou K, Detels R. Prevalence of HIV infection among former commercial plasma donors in rural eastern China. Health Policy Plan. 2001;16(1):41-6.

25. Liu Z, Mei Z, Zheng X, Zhang W, Wang F, Zhou Y, et al. Investigation of HIV infection among donors who engaged in three underground plasma collection events in Central China. Chinese Journal of Epidemiology. 2000;21(6):466-7.

26. Qian HZ, Vermund SH, Kaslow RA, Coffey CS, Chamot E, Yang $\mathrm{Z}$, et al. Co-infection with HIV and hepatitis $\mathrm{C}$ virus in former plasma/blood donors: challenge for patient care in rural China. AIDS. 2006;20(10):1429-35.

27. Ministry of Health of the People's Republic of China, Joint United Nations Programme on HIV/AIDS, World Health Organization. 2005 update on the HIV/AIDS epidemic and response in China. Beijing: National Center for AIDS/STD Prevention and Control, China CDC; 2005.

28. Xiao Y, Kristensen S, Sun J, Lu L, Vermund SH. Expansion of HIV/ AIDS in China: lessons from Yunnan Province. Soc Sci Med. 2007;64(3):665-75.

29. Chu TX, Levy JA. Injection drug use and HIV/AIDS transmission in China. Cell Res. 2005;15:865-9.

30. Zhang J, Cheng H, Duan S, Duan Y, Zhao S, Wang S, et al. Survey on the current situation of epidemic of HIV infection in Yunnan Province. Chinese Journal of Epidemiology. 1994;15(5):259-62.

31. Sullivan SG, Wu Z. Rapid scale up of harm reduction in China. Int $\mathbf{J}$ Drug Policy. 2007;18(2):118-28.

32. Yan C, Liau A, Wu Z. An overview of the history of epidemic of and response to HIV/AIDS in China: achievements and challenges. Chin Med J. 2009;122(19):2251-7.
33. Wang L, Guo W, Li D, Ding Z, McGoogan J, Wang N, et al. HIV epidemic among drug users in China: 1995 to 2011. Addiction. 2015;110(1):20-8.

34. National Health and Family Planning Commission of the People's Republic of China. 2015 China AIDS response Progress report. Beijing: Chinese Health and Family Planning Commission; 2015.

35. Chen Q, Sun Y, Sun W, Hao M, Li G, Su X, et al. Trends of HIV incidence and prevalence among men who have sex with men in Beijing, China: nine consecutive cross-sectional surveys, 20082016. PLoS One. 2018;13(8):e0201953.

36. OutRight Action International. China: The legal position and status of lesbian, gay, bisexual and transgender people in the People's Republic of China Beijing2010 [updated Mar 24, 2010. Available from: https://www.outrightinternational.org/content/china-legalposition-and-status-lesbian-gay-bisexual-and-transgender-peoplepeople\%E2\%80\%99s.

37. Jia Y, Aliyu MH. Huang ZJ. BioMed Research International: Dynamics of the HIV Epidemic in MSM; 2014.

38. Sun X, Xiao Y, Peng Z, Wang N. Frequent implementation of interventions may increase HIV infections among MSM in China. Sci Rep. 2018;8:451.

39. Cui Y, Liau A, Wu ZY. An overview of the history of epidemic of and response to HIV/AIDS in China: achievements and challenges. Chin Med J. 2009;122(19):2251-7.

40. Wu Z. Characteristics of HIV sexually transmission and challenges for controlling the epidemic in China. Zhonghua Liu Xing Bing Xue Za Zhi. 2018;39(6):707-9. This paper provides an updated account of the charaterisics of HIV sexual transmission and the many challenges China faces in controlling its HIV/AIDS epidemic.

41. He N, Duan S, Ding Y, Rou K, McGoogan JM, Jia M, et al. Antiretroviral therapy reduces HIV transmission in discordant couples in rural Yunnan. China PLoS One. 2013;8(11):e77981.

42. National Center for AIDS/STD Control and Prevention, China CDC. China National HIV/syphilis/HCV Surveillance Report in 2015. Beijing: National Center for AIDS/STD control and prevention, China CDC; 2015.

43. Shan $\mathrm{D}, \mathrm{Yu} \mathrm{MH}$, Yang J, Zhuang $\mathrm{MH}$, Ning Z, Liu H, et al. Correlates of HIV infection among transgender women in two Chinese cities. Infect Dis Poverty. 2018;7(1):123.

44. Best J, Tang W, Zhang Y, Han L, Liu F, Huang S, et al. Sexual behaviors and HIV/syphilis testing among transgender individuals in China: implications for expanding HIV testing services. Sex Transm Dis. 2015;42(5):281-5.

45. Yang X, Zhao L, Wang L, Hao C, Gu Y, Song W, et al. Quality of life of transgender women from China and associated factors: a cross-sectional study. J Sex Med. 2016;13:977-87.

46. Zeng H, Chow EP, Zhao Y, Wang Y, Tang M, Li L, et al. Prevention of mother-to-child HIV transmission cascade in China: a systematic review and meta-analysis. Sex Transm Infect. 2016;92(2):116-23.

47. Jiang JR, Zhou YB, Li HT, Gao YQ, Zhang YL, Luo SS, et al. HIV epidemic among pregnant women in China, 2016: trend and spatial analysis. Zhonghua Yi Xue Za Zhi. 2018;98(41):3360-4.

48. Wang A, Qiao Y, Dou L, Wang Q, Wang X, Su M, et al. Challenges of eliminating mother-to-child transmission of HIV, syphilis and hepatitis B in China: a cross-sectional survey. Lancet. 2018;392: S55.

49. Wu Z, Rou K, Cui H. The HIV/AIDS epidemic in China: history, current strategies and future challenges. AIDS Educ Prev. 2004;16(3 Suppl A):7-17.

50. Yang S, Wu J, Ding C, Cui Y, Zhou Y, Li Y, et al. Epidemiological features of and changes in incidence of infectious diseases in China in the first decade after the SARS outbreak: an observational trend study. Lancet Infect Dis. 2017;17(7):716-25.

51. Information Office, Ministry of Health. 2003 Notifiable diseases in China. Bulletin of Ministry of Health 2004:63-64. 
52. Information Office, Ministry of Health. Ministry of Health releases notifiable diseases in China in January 2009 And in 2008. Bulletin of Ministry of Health. 2009:65-67.

53. Bureau of Disease Control, National Health Commission of the People's Republic of China. Summary of 2018 notifiable diseases in China Beijing: National Health Commission of the People's Republic of China; 2019 [.
54. Gao D, Zou Z, Dong B, Zhang W, Chen T, Cui W, et al. Secular trends in HIV/AIDS mortality in China from 1990 to 2016: gender disparities. PLoS One. 2019;14(7):e0219689.

Publisher's Note Springer Nature remains neutral with regard to jurisdictional claims in published maps and institutional affiliations. 